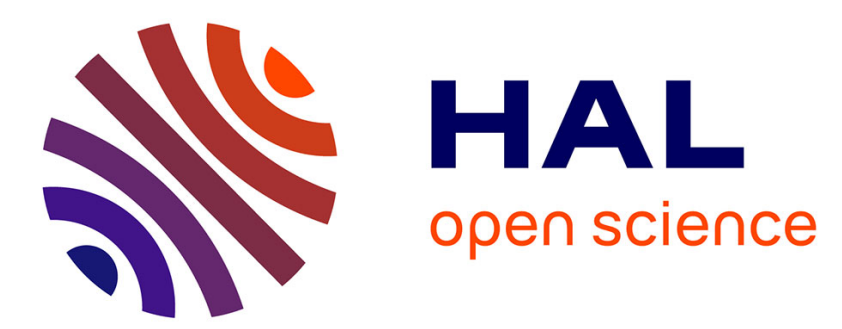

\title{
Eating Rewards the Gears of the Clock
}

Jorge Mendoza

\section{To cite this version:}

Jorge Mendoza. Eating Rewards the Gears of the Clock. Trends in Endocrinology and Metabolism $=$ Trends in Endocrinology \& Metabolism , 2019, 30 (5), pp.299-311. 10.1016/j.tem.2019.03.001. hal-02386636

\section{HAL Id: hal-02386636 \\ https://hal.science/hal-02386636}

Submitted on 22 Oct 2021

HAL is a multi-disciplinary open access archive for the deposit and dissemination of scientific research documents, whether they are published or not. The documents may come from teaching and research institutions in France or abroad, or from public or private research centers.
L'archive ouverte pluridisciplinaire HAL, est destinée au dépôt et à la diffusion de documents scientifiques de niveau recherche, publiés ou non, émanant des établissements d'enseignement et de recherche français ou étrangers, des laboratoires publics ou privés.

\section{다)(1) $(5$}

Distributed under a Creative Commons Attribution - NonCommercial| 4.0 International 
$\underline{2}$

$\underline{3}$

$4 \quad$ Jorge Mendoza*

$\underline{5}$ Institute of Cellular and Integrative Neurosciences, CNRS UPR-3212, 5 rue Blaise Pascal,

6 67000 Strasbourg, France

$\underline{7}$

$\underline{8}{ }^{*}$ Corresponding Author:

$\underline{9} 5$ rue Blaise Pascal

1067000 Strasbourg

$11 \quad$ Tel 03.88.45.66.96

12 jmendoza@inci-cnrs.unistra.fr

13 ORCID (JM): 0000-0003-2819-9624

$\underline{14}$

$\underline{15}$

Keywords: circadian; eating; hedonic; metabolic; suprachiasmatic; clock genes 
35 Eating behavior is regulated by metabolic and hedonic brain networks which interact each other to balance the physiological regulation of hunger and satiety. The daily balance this regulation is controlled by the central circadian clock. Importantly, metabolic and reward properties of food impact the functioning of circadian clocks, altering the oscillatory activity of the molecular clockwork and circadian rhythms. However, when feeding (metabolic or reward) is timed the whole circadian system is entrained. Furthermore, besides synchronizing the clock, the timing of both metabolic and reward eating might be crucial for health: to both improve circadian physiology as well as to treat metabolic (diabetes, obesity) and neurological diseases (mental, neurodegenerative). 
Food intake is a complex behavior that involves the participation of neural substrates that function, in an interactive and balanced manner, in conjunction with peripheral physiology in various organs that indicate the energy state of the organism, in both hunger and satiety.

The classical mechanisms of the regulation of food intake involve two principal systems in the brain [1]: the first, a metabolic system that regulates the amount of food necessary for the survival of the organism, and a hedonic or pleasure mechanism that regulates the quality and type of food selected and ingested [1]. While the metabolic mechanisms are mainly regulated by the hypothalamus and brain stem, the hedonic processes are principally, but not uniquely, regulated by the dopamine (DA) system. Anatomically and functionally these centers in the brain are linked, and this gives a balanced regulation between the quantity and quality of what has to be eaten. In addition, food-related hormones (e.g., insulin, leptin and ghrelin) have the ability to modulate the cellular activity of brain nuclei of both systems due to the presence of specific receptors. Therefore, the physiological regulation of food intake depends on the homeostatic interaction between the brain and the periphery. When this homeostasis is lost, metabolic pathologies appear.

Since physiology is clock-controlled, food intake must be regulated in time. Therefore, centrally the circadian clock coordinates the time of metabolic and hedonic food intake, influencing the quality and quantity of food to be taken at a specific time of the day [2]. On the other hand, the timing of food intake can either positively (entrainment) or negatively (misalignment) affect the circadian system. Furthermore, these effects may be even more evident when particular types of diets are eaten (e.g., high fat, hypocaloric, palatable diets).

\section{Time Eating Behavior: paced by the $\operatorname{clock}(\mathrm{s})$}

Food intake is a robust daily rhythm accompanying increased locomotor activity around the event. Mammalian diurnal species, including humans, distribute food intake across the day or light phase of the light-dark (LD) cycle imposed by the solar time [3, 4]. While nocturnal rodents, eat higher amounts of food in the early night with a second peak of feeding in the late night [5]. Essentially, daily variations of eating patterns are synchronized to the LD cycle through the suprachiasmatic (SCN) circadian pacemaker (and their molecular clockwork; Box 1) which receives direct photic information from the retina (Box 2), and transmits this to brain structures that regulate feeding $[2,6]$ (Figure 1).

The SCN projects to the metabolic hypothalamus via the subparaventricular zone which then distributes timing information to the paraventricular (PVN), dorsomedial (DMH) and lateral 
hypothalamus (LH) [7] (Figure 1). Cells in the LH express the neuropeptides orexins (ORX) which are involved in locomotion and feeding behaviors and show daily (synchronized to the 24h LD cycle) and circadian (self-sustained under constant light conditions) expression at both the gene and protein level. ORX release in nocturnal rodents peaks at the beginning of the night, the time at which the animals are awake and begin feeding $[8,9]$. Moreover, ORX have also been implicated in arousal and reward related to feeding [10]. Thus, the SCN communicates to the LH-ORX neurons to initiate rhythms of locomotion and metabolichedonic eating.

The SCN also provides timing information to brain regions regulating the hedonic drive of eating (Figure 1). In the midbrain the DAergic ventral tegmental area (DA-VTA) projects to the nucleus accumbens (NAcc) in the ventral striatum for the regulation of motivated behaviors $[11,12]$. In the NAcc medium spiny GABAergic neurons contain DA receptors (D1 and D2 type) which are activated when eating, and this response is still higher if a palatable diet is ingested, leading to reinforcement of this behavior. Moreover, the DA tone in VTA cells might be modulated by metabolic factors such as ghrelin and leptin to promote and inhibit, respectively, reward eating $[12,13]$.

DA release and content in the striatum is rhythmic, and in nocturnal rats and mice higher levels occur at night, and in the day for diurnal rodents: the time of locomotion and feeding [14-16]. Thus, DA rhythms are likely participating in the modulation of locomotion and feeding rhythms. Anatomically, the SCN projects to the VTA indirectly via the medial preoptic nucleus (MPON) of the hypothalamus [17]; thence, VTA-DA circadian rhythms related to feeding might be induced or at least regulated by the $\mathrm{SCN}$; at first, to initiate locomotion at the right time for food-seeking behaviors and secondly to modulate circadian food-reward at night [18] or day in nocturnal or diurnal species, respectively. This last point might be crucial to facilitate and promote reinforcement and conditioned learning associated to food intake.

Accordingly, the control of food intake in the brain is dependent on this balanced MetabolicHedonic-Circadian circuit [2]. However, when one of these components is out of balance food intake is altered, often leading to overconsumption and weight gain. The exposure to and intake of high caloric diets induces several changes in all these brain centers regulating feeding, including those from the circadian system.

The ad libitum intake of high caloric diets leads to alterations in circadian rhythms of behavior and on the expression of clock genes and those regulating energy balance in the 
hypothalamus and peripheral tissues [19]. Furthermore, the behavioral and molecular entrainment to light is affected in mice fed with a high fat diet [20]. Taken together, these results indicate that high-fat feeding alters the functioning and light synchronization of the mammalian circadian clock.

In an experimental model in which rodents were given the choice between a high-caloric diet vs. a normocaloric diet, animals show "snacking" feeding behavior and preference for the caloric diet along the whole $24 \mathrm{~h} \mathrm{LD}$ cycle, disrupting thus the daily rhythm of eating and leading in rapid gain weight $[21,22]$. Thus, beyond the type of diet, the loss of daily rhythms of feeding becomes an important factor in inducing obesity and metabolic diseases.

Centrally, in animals exposed to the protocol of diet choice, the day-night expression of the clock proteins PER2 and BMAL1 in the mouse lateral habenula (LHb) is disrupted by the intake of the caloric diet [21]. The LHb is an epithalamic nucleus which is relevant for the control of monoamines and the regulation of palatable food intake [23, 24]. Interestingly, sleep deprivation, a condition that increases caloric intake and body weight gain [25], disrupts clock gene expression in the LHb [26], while a perturbation of the LHb clock by high caloric diets might lead to disruption of the sleep-wake cycle. This highlights a specific circadian role of the LHb in rhythmic caloric intake, likely via its control on DAergic hedonic centers [27]. Accordingly, it was recently reported that rhythms of DA release in the SCN are disrupted in an animal model of obesity [28]. Therefore, DA rhythms in the SCN and the rest of the forebrain could be necessary to maintain a healthy metabolic condition and proper food intake.

\section{Time Eating Behavior: pacing clock(s)}

\section{Time-Restricted Eating, a good or bad clock aligner? A matter of time}

Time-restricted eating (TRE) induces an increase in the locomotor activity pattern in anticipation to meal access and the synchronization of physiological rhythms and metabolic activity, indicating that feeding time is a strong synchronizer for circadian rhythms $[29,30]$ (Figure 2A). Nevertheless, the SCN (clock gene expression) remains entrained to the LD cycle, thus TRE uncouples rhythms of clock genes in peripheral [31] (liver, lung) (Box 3) and extra-SCN [32] (hypothalamic and limbic) oscillators (Box 4) from the main SCN pacemaker. However, the uncoupling of daily feeding rhythms from the LD cycle can lead to negative metabolic consequences. Actually, in laboratory animals, mainly mice and rats, TRE has been imposed in the light phase (when nocturnal rodents sleep) which perturbs their sleep-wake 
cycle. Metabolically, when mice are fed with a high-fat diet only during the light phase of the LD cycle, body weight increase is significantly higher than in animals fed at the dark phase [33].

Contrary, TRE at night, when nocturnal rodents are active, leads to diverse effects in metabolism and physiology, improving circadian rhythms even in clock gene mutant mice [34-36]. TRE protects against obesity induced by diet, in double global Cryl-2 mutants and in mice carrying a liver-specific deletion of Bmall and/or Rev-Erb $\alpha / \beta$. More importantly, TRE at night effects against obesity, hyperinsulinemia and inflammation are observed in animals fed with a high caloric diet, even if these eat the same amount of calories as that exposed $a d$ libitum to the diet. In both clock altered mice and/or those exposed to hypercaloric diets, TRE at night modifies gene rhythms in the liver, permitting better energy utilization and expenditure, and avoiding the development of obesity or metabolic syndrome [34, 35]. Thus, TRE might have both beneficial or negative consequences in physiology; the difference is in the time of feeding aligned with the sleep-wake cycle [33] (Figure 2A).

What are the signals by which TRE entrains peripheral clocks? Body temperature, hormones and peptides related to feeding are important candidate signals for the entrainment of peripheral oscillators, and even circadian clocks in extra-SCN brain sites [37]. Recently, it was reported that oxyntomodulin (OXM) is the principal signal, induced by feeding, that entrains the circadian clock in the liver through activation of Per1 [38]. Importantly, the effect of OXM on the liver is clock-dependent. When mice are treated with OXM at day the liver clock shifts, but not when OXM is given at night [38]. It will be interesting to determine whether OXM can entrain other peripheral oscillators, and to identify new peripheral or central signals that specifically entrain peripheral and central oscillators by feeding time (Figure 2A).

\section{Time-restricted eating for the master clock: a matter of time and calories}

Circadian rhythms of locomotion, body temperature, hormonal release (all of them SCNoutputs), and SCN clock gene expression are phase-shifted or entrained when TRE is coupled to a caloric restriction (CR) in nocturnal and diurnal rodents. This suggests that in addition to feeding time, a caloric condition promotes resetting of the SCN circadian clock $[4,39]$.

CR has beneficial effects in health and lifespan through the activation of the sirtuin 1 (SIRT1) and the mitochondrial oxidative metabolism [40]. Interestingly, at the clock level, SIRT1 regulates the circadian rhythms of peripheral tissues and central structures, having as 
molecular targets BMAL1 and PER2 clock components, deacetylating both proteins [41]. Thus, changing the cellular metabolic state of the SCN, CR can entrain the molecular clockwork (Figure 2B).

On the other hand, SIRT1 in the VMH is relevant for the control of energy balance, mainly sensing feeding and metabolic cues. The VMH projects directly to the SCN, thus CR might induce the expression of SIRT1 in the VMH, and then, indirectly, alter the molecular timekeeping in the SCN [42]. However, as the SCN clock receives direct projections from other hypothalamic and non-hypothalamic structures related feeding, it is highly likely that entrainment of the SCN by feeding is dependent on several inputs from the whole brain.

\section{Time-palatable eating: Rewarding the clock}

Food intake induces the activation of the regions of the hedonic reward system. The intake of palatable diets triggers the release of DA from the VTA in the midbrain to the ventral NAcc [12]. Moreover, DA release might also target other brain sites containing DA receptors such as the prefrontal cortex, the striatum and, importantly, the SCN [43]. The VTA projects a functional pathway directly to the SCN, targeting D1-type receptors in clock cells to facilitate the re-entrainment of behavioral rhythms to a new LD cycle [43]. Activation of DA receptors leads to intracellular changes in the mitogen-activated protein kinase (MAPK) and cAMP response element-binding protein (CREB), and finally an increase in the expression of $\mathrm{Per}$ genes [44]. Then, DA release by feeding time can induce Per gene expression in the SCN clock via these pathways (Figure 2B). Furthermore, when feeding is highly palatable, DA release is enhanced having a major impact in the control of behavior (e.g., learning, reinforcement), including circadian behavior (synchronization) $[12,45]$.

In animals fed with a daily palatable diet, without food restriction, circadian rhythms of locomotor activity (Figure 2C) and clock gene expression are synchronized to the time of feeding $[45,46]$. Moreover, in arrhythmic Per mutant mice a daily palatable snack is able to rescue and entrain behavioral rhythms [47]. Altogether, the effects of daily palatable meal to entrain and rescue circadian rhythms are certainly dependent, in part, on DA activity. DA release at the level of the forebrain (e.g., striatum, NAcc, SCN) is rhythmic [14, 28], and synchronized by feeding (palatable or regular diet); hence, feeding time can reach SCN clock (via D1 receptors) inducing the synchronization of molecular, behavioral and physiological rhythms (Figure 2A-C). However, the neural mechanisms of palatable food entrainment become more complex when we add other systems, substrates and molecules participating in the regulation of hedonic feeding and that can affect the SCN. 
Serotonin (5-HT) from the raphe nuclei is an important internal synchronizer for the SCN since the clock contains diverse types of 5-HT receptors $\left(5-\mathrm{HT}_{1 \mathrm{~A}, 1 \mathrm{~B}}, 2 \mathrm{~A}, 2 \mathrm{~B}\right.$ and 7$)$. 5-HT has been implicated in the non-photic synchronization of the clock via $5-\mathrm{HT}_{1 \mathrm{~A}}$ and $5-\mathrm{HT}_{7}$ receptors, the induction of both the protein kinasa $\mathrm{A}(\mathrm{PKA})$ and $\mathrm{K}^{+}$channels, and changes in Per gene expression [48]. Intake of meals rich in carbohydrates increases hypothalamic 5-HT release, and 5-HT neurons in the raphe nuclei show c-Fos expression in animal synchronized to daily palatable meals $[46,49]$. Thus, 5 -HT may be involved in the food-reward entrainment of the SCN clock (Figure 2B).

ORX are able to shift molecular and electrical activity of the SCN through the activation of ORX -1 and 2 receptors, changing intracellular $\mathrm{Ca}^{2+}$ levels and leading in a suppression of Per genes [50]. In the entrainment of circadian rhythms and molecular clock gene expression in the SCN by daily access to a palatable feeding, ORX cells are significantly activated (c-Fos expression) [46]. In addition, a molecular disruption of the clock, which leads to compulsive intake of palatable food, upregulates ORXergic activity [9]. Thus, ORX are another internal time cue with the ability to entrain the clock when palatable feeding is timed by changing Per gene expression (Figure 2B).

Endocannabinoids are involved in the central regulation of feeding at both levels, metabolic and hedonic [51]. 2-arachidonoylglycerol (2-AG) and N-arachidonylethanolamine (AEA), the two principal ligands of the endocannabinoids receptors (CB1 and CB2) show circadian variations in the brain, and CB1 receptors are expressed in the SCN nuclei $[52,53]$. CB1 receptors in the SCN presynaptically regulate GABAergic transmission; thus, cannabinoids upregulate the firing rate of SCN cells and modulate the light entrainment of the clock by a presynaptic mechanism [53]. Therefore, during palatable feeding time, CB might impact the SCN clock as an entraining signal via the modulation of the GABAergic neurotransmission and clock gene expression (Figure 2B).

Similarly, the opiod-circuitry, implicated in food-reward related behaviors, might affect the circadian system [54]. Motivation for the intake of palatable foods is modulated by opioid (enkephalin) action in the forebrain. $\mu$ opioid receptors are contained in the forebrain including in the SCN, which is reset by selective agonists of these receptors via the ERK1/2 and GSK3 $\beta$ signaling pathways $[54,55]$. Thus, opioid signals rising after palatable feeding might be able to time code the SCN to potentiates and induce entrainment to timed food reward. 
Additionally, from the periphery the metabolic-reward hormones, leptin and ghrelin, may impact the SCN to reinforce or coordinate entrainment to feeding time. In vitro, both leptin and ghrelin reset the circadian electrical and clock gene activity in the SCN [56, 57], likely via direct actions on leptin and ghrelin receptors, respectively [58, 59]; hence, in time restricted eating, high levels of ghrelin and leptin after and before feeding, respectively, are important physiological signals for the entrainment of circadian clocks bolstering the brain metabolic and hedonic drives of feeding for SCN synchronization (Figure 2B).

In summary, when the clock is entrained to food with a strong palatable and reward content, several brain circuits give timing information to the SCN. The summation and temporal coordination (before, during and after feeding) of these might lead to the entrainment of the clock and the expressed behavioral and physiological rhythms.

\section{Circadian food entrainment in humans: physiology, behavior and gene expression.}

The knowledge on the effects of food entrainment on the circadian physiology of rodents has advanced profoundly, highlighting that feeding time synchronizes behavior, physiology (hormonal rhythms) and gene expression in the brain, including the SCN and peripheral tissues.

However, there is still much to be understood on the effects of meal timing on the human circadian system and health. This becomes a subject of scientific and societal interest due to the increasing epidemic of metabolic diseases worldwide such as obesity and diabetes, but also due to eating disorders (e.g., compulsive feeding, binge eating, night eating disorder).

In healthy conditions, human feeding behavior follows a daily and circadian rhythm [3]; however, this may be disrupted under particular conditions of overeating, malnutrition or by shifting the time of feeding. Accordingly, calorie intake at different times of day differentially impacts energy balance and metabolism [60]. Humans, being a diurnal species, have to distribute calorie intake mainly in the early day and reduce it at night. Unfortunately, this is not the current situation in human eating behavior. Healthy subjects without a phase shift condition (e.g., shift work, jet-lag) show aberrant eating behavior with a principal caloric intake at night and less at day [61].

Similar to what has been reported in rodents, when mealtime is out of phase with the sleep wake cycle, the human circadian system is in a state of internal misalignment between central and peripheral clocks, which could lead to pathology (e.g., obesity, diabetes) [62]. Previous 
studies have reported that eating late at night increases the risk of obesity. In addition, the risk is higher still when eating earlier in the day (breakfast) is omitted [63].

Certain social conditions in which people are exposed to circadian disruptions such as shift work or social jet-lag lead to an increased calorie intake at night, which is significantly correlated with obesity $[64,65]$. Calorie intake at night leads to clock gene expression changes in peripheral oscillators (adipose tissue), hormonal release (leptin, insulin) and changes in plasma glucose rhythms and glucose tolerance, which altogether may induce increased appetite and food intake [66]. In shift work conditions, the daily oscillations of the hunger hormone ghrelin are shifted with a postprandial increase which might trigger (via VTA-DAergic neurons) hedonic eating at night [67].

Contrary to this, when feeding happens at early times of day physiological responses are different. Gastrointestinal absorption is higher during the day, thus calorie intake at day improves glucose tolerance and plasma insulin levels during fasting conditions. Moreover, feelings of hunger are reduced when calorie intake is principally in the day than at night [60]. Importantly, a group of subjects with prediabetes exposed to temporal restricted eating during the day, showed improvements in insulin sensitivity, $\beta$ cell responsiveness, blood pressure and appetite, indicating the beneficial effects, even in a pathological condition, of timerestricted eating early at day [68].

Timing of caloric intake affects brain DA and 5-HT metabolism in both rodents and humans [12]. In obese subjects, caloric intake in the early morning increases the binding of the DA (DAT) and serotonin (SERT) transporters in the ventral striatum and thalamus respectively. This effect was diminished when the subjects' caloric intake was higher at night (dinner) [69]. A reduction of the dopamine D2 receptors availability in the striatum has been associated with body weight increase and obesity [70]. DA cell activity shows daily variations, hence, the effects of mealtime in the day on DA metabolism might be dependent on the rhythmic properties of the DAergic system. Moreover, hormonal changes (leptin and ghrelin) by timed calorie intake at the early morning could potentiate DA metabolism and contribute to reduced food intake and body weight.

Altogether, the important data on the effects of food entrainment in the human circadian system and metabolism indicate that a timed food intake aligned with the sleep-wake cycle and other hormonal rhythms improves and maintains healthy physiology. In mealtime 
synchronization both central systems controlling eating (metabolic and hedonic) are importantly implicated.

\section{$\underline{318}$}

$\underline{319}$

$\underline{320}$

$\underline{321}$

$\underline{322}$

$\underline{323}$

$\underline{324}$

\section{Conclusions and future perspectives}

In the present review some of the most important, but unfortunately not all, discoveries of the effects of feeding behavior on circadian rhythms and clocks have been highlighted, as well as how food intake affects the main clock in the SCN when the caloric content and/or the hedonic value of the food change. The neural and molecular mechanisms of food entrainment of circadian clocks are not yet fully described; however, important advances indicate that the central pathways underlying food intake (metabolic and hedonic) must be involved.

However, there are still several questions regarding the mechanisms of entrainment of peripheral oscillators and the SCN clock to food timing with or without palatable diets. For example, how do reward components of palatable diets (e.g., sucrose, fat) reach the brain and then the SCN? Are these effects clock-dependent? If so, what are the optimal times to affect the SCN clock by feeding? Are clock genes implicated in SCN food entrainment?

The timing of eating has an important clinical application in human health. Thus, new proposals in research are necessary to understand the central and peripheral (circadian and non-circadian) mechanisms that underlie the effects of meal timing in physiology, and how the principal SCN clock, which was neglected as a "food-entrainable oscillator", is entrained by the time of eating.

What is the relevance of knowing these mechanisms? If we determine how feeding time synchronizes the clock in the SCN, it would be feasible to think that "feeding-time therapy" can be used in chronobiology to treat circadian and non-circadian pathologies in which the oscillatory capacity of the clock is compromised.

When the SCN clock is genetically altered, this becomes highly sensitive and responsive to time feeding-metabolic-hedonic cues, even more than to light-dark cycles [71-73]. Thus, in circadian pathological conditions (e.g., shift-work, social jet-lag, seasonal depression) in which light has been used as treatment, feeding time might replace it as a "new medication" to rescue SCN circadian rhythms. Moreover, it will not be surprising or unreasonable to think that food entrainment can be used to treat obesity, diabetes, eating disorders [74], and also other mental illnesses such as depression or neurodegenerative diseases (e.g., Parkinson, Alzheimer, Huntington disease) [72] in which a disturbed clock has to be readjusted. 
Further, even more interesting is the proposition of combine light and feeding therapies to set in time circadian clocks and improve health. For this, it is first necessary to understand the putative circadian and brain mechanisms shared by light and food to potentiate and facilitate the entrainment of the circadian system.

\section{Acknowledgements}

I would like to thank Marie-Paule Felder-Schmittbuhl, Wilf Gardner and all the members of my group (Circadian-Reward) for their constructive comments and discussions. I am gratefully acknowledged to the Agence National de la Recherche (ANR-14-CE13-0002-01 ADDiCLOCK JCJC), the consortium Danone/Foundation pour la Recherche Medicale (FRM) and the Centre National de la Recherche Scientifique (CNRS) for funding. I do apologize to all my colleagues whose relevant contributions could not be cited due to space limitations.

\section{References}

1. Rossi, M.A. and G.D. Stuber, Overlapping Brain Circuits for Homeostatic and Hedonic Feeding. Cell Metab, 2018. 27(1): p. 42-56.

2. Mendoza, J.., Food intake and addictive-like eating behaviors: Time to think about the circadian clock(s). Neurosci Biobehav Rev, 2018.

3. de Castro, J.M., Circadian rhythms of the spontaneous meal pattern, macronutrient intake, and mood of humans. Physiol Behav, 1987. 40(4): p. 437-46.

4. Mendoza, $\underline{\text { J. }}$ et al., Setting the main circadian clock of a diurnal mammal by hypocaloric feeding. J Physiol, 2012. 590(13): p. 3155-68.

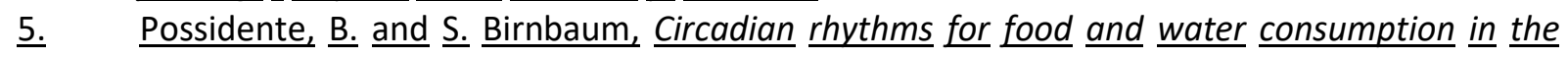
mouse, Mus musculus. Physiol Behav, 1979. 22(4): p. 657-60.

6. Patton, A.P. and M.H. Hastings, The suprachiasmatic nucleus. Curr Biol, 2018. 28(15): p. R816-R822.

7. Vujovic, N., et al., Projections from the subparaventricular zone define four channels of output from the circadian timing system. J Comp Neurol, 2015. 523(18): p. 2714-37.

8. Deboer, $\mathrm{T}_{.}$et al.., Convergence of circadian and sleep requlatory mechanisms on hypocretin1. Neuroscience, 2004. 129(3): p. 727-32.

9. Feillet, C.A., et al., Rev-erbalpha modulates the hypothalamic orexinergic system to influence pleasurable feeding behaviour in mice. Addict Biol, 2017. 22(2): p. 411-422.

10. Sakurai, T., The role of orexin in motivated behaviours. Nat Rev Neurosci, 2014. 15(11): p. 719-31.

11. Morales, M. and E.B. Margolis, Ventral tegmental area: cellular heterogeneity, connectivity and behaviour. Nat Rev Neurosci, 2017. 18(2): p. 73-85.

12. Volkow, N.D., R.A. Wise, and R. Baler, The dopamine motive system: implications for druq and food addiction. Nat Rev Neurosci, 2017. 18(12): p. 741-752.

13. Liu, S. and S.L. Borgland, Regulation of the mesolimbic dopamine circuit by feeding peptides. Neuroscience, 2015. 289: p. 19-42.

14. Hood, S., et al., Endogenous dopamine regulates the rhythm of expression of the clock protein $\underline{P E R 2}$ in the rat dorsal striatum via daily activation of $\underline{D 2}$ dopamine receptors. $\mathrm{J}$ Neurosci, 2010. 30(42): p. 14046-58.

15. Ferris, M.J., et al., Dopamine transporters govern diurnal variation in extracellular dopamine tone. Proc Natl Acad Sci U S S A, 2014. 111(26): p. E2751-9. 
16. Itzhacki, J., et al., Light rescues circadian behavior and brain dopamine abnormalities in diurnal rodents exposed to a winter-like photoperiod. Brain Struct Funct, 2018. 223(6): p.

17. Luo, A.H. and G. Aston-Jones, Circuit projection from suprachiasmatic nucleus to ventral tegmental area: a novel circadian output pathway. Eur J Neurosci, 2009. 29(4): p. 748-60.

18. Bainier, $\underline{C}$., et al., Circadian rhythms of hedonic drinking behavior in mice. Neuroscience, 2017. 349: p. 229-238.

19. Kohsaka, A., et al., High-fat diet disrupts behavioral and molecular circadian rhythms in mice. Cell Metab, 2007. 6(5): p. 414-21.

20. Mendoza, J.. P. Pevet, and E. Challet, High-fat feeding alters the clock synchronization to light. J Physiol, 2008. 586(24): p. 5901-10.

21. Blancas-Velazquez, A., S.E. la Fleur, and J. Mendoza, Effects of a free-choice high-fat highsugar diet on brain PER2 and BMAL1 protein expression in mice. Appetite, 2017. 117: p. 263$\underline{269}$.

22. $\underline{\text { la Fleur, }}$ S.E., et al., The snacking rat as model of human obesity: effects of a free-choice highfat high-sugar diet on meal patterns. Int $\mathrm{J}$ Obes (Lond), 2014. 38(5): p. 643-9.

23. Mizumori, S.J.Y. and P.M. Baker, The Lateral Habenula and Adaptive Behaviors. Trends Neurosci, 2017. 40(8): p. 481-493.

24. Stamatakis, A.M., et al., Lateral Hypothalamic Area Glutamatergic Neurons and Their Projections to the Lateral Habenula Regulate Feeding and Reward. J Neurosci, 2016. 36(2): p. 302-11.

25. Dashti, H.S., et al., Short sleep duration and dietary intake: epidemiologic evidence, mechanisms, and health implications. Adv Nutr, 2015. 6(6): p. 648-59.

26. Zhang, B., et al., Sleep Deprivation Influences Circadian Gene Expression in the Lateral Habenula. Behav Neurol, 2016. 2016: p. 7919534.

27. Mendoza, J., Circadian neurons in the lateral habenula: Clocking motivated behaviors. Pharmacol Biochem Behav, 2017.

28. Luo, S., et al., Circadian peak dopaminergic activity response at the biological clock pacemaker (suprachiasmatic nucleus) area mediates the metabolic responsiveness to $\underline{\text { a high- }}$ fat diet. J Neuroendocrinol, 2018. 30(1).

29. Patton, D.F. and R.E. Mistlberger, Circadian adaptations to meal timing: neuroendocrine mechanisms. Front Neurosci, 2013. 7: p. 185.

30. Mendoza, J., Circadian clocks: setting time by food. J Neuroendocrinol, 2007. 19(2): p. 12737.

31. Damiola, $F_{.}$et al., Restricted feeding uncouples circadian oscillators in peripheral tissues from the central pacemaker in the suprachiasmatic nucleus. Genes Dev, 2000. 14(23): p. 2950-61.

32. Verwey, M. and S. Amir, Food-entrainable circadian oscillators in the brain. Eur $\underline{J}$ Neurosci, 2009. 30(9): p. 1650-7.

33. Arble, D.M., et al., Circadian timing of food intake contributes to weight gain. Obesity (Silver Spring), 2009. 17(11): p. 2100-2.

34. Chaix, A., et al., Time-Restricted Feeding Prevents Obesity and Metabolic Syndrome in Mice Lacking a Circadian Clock. Cell Metab, 2018.

35. Hatori, M., et al., Time-restricted feeding without reducing caloric intake prevents metabolic diseases in mice fed a high-fat diet. Cell Metab, 2012. 15(6): p. 848-60.

36. Chaix, A., et al., Time-restricted feeding is a preventative and therapeutic intervention against diverse nutritional challenges. Cell Metab, 2014. 20(6): p. 991-1005.

37. Schibler, U., et al., Clock-Talk: Interactions between Central and Peripheral Circadian Oscillators in Mammals. Cold Spring Harb Symp Quant Biol, 2015. 80: p. 223-32.

38. Landgraf, D., et al., Oxyntomodulin requlates resetting of the liver circadian clock by food. Elife, 2015. 4: p. e06253. 
39. Mendoza, J., et al., Feeding cues alter clock gene oscillations and photic responses in the

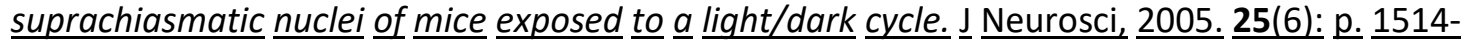
22.

40. Libert, S. and L. Guarente, Metabolic and neuropsychiatric effects of calorie restriction and sirtuins. Annu Rev Physiol, 2013. 75: p. 669-84.

41. Masri, S., et al., Coupling circadian rhythms of metabolism and chromatin remodelling. Diabetes Obes Metab, 2015. 17 Suppl 1: p. 17-22.

42. Orozco-Solis, R., et al., SIRT1 Relays Nutritional Inputs to the Circadian Clock Through the Sf1 Neurons of the Ventromedial Hypothalamus. Endocrinology, 2015. 156(6): p. 2174-84.

43. Grippo, R.M., et al., Direct Midbrain Dopamine Input to the Suprachiasmatic Nucleus Accelerates Circadian Entrainment. Curr Biol, 2017.

44. Akashi, M. and $\mathrm{E}$. Nishida, Involvement of the MAP kinase cascade in resetting of the mammalian circadian clock. Genes Dev, 2000. 14(6): p. 645-9.

45. Mendoza, J., M. Angeles-Castellanos, and C. Escobar, $\underline{A}$ daily palatable meal without food deprivation entrains the suprachiasmatic nucleus of rats. Eur J Neurosci, 2005. 22(11): p. 2855-62.

46. Mendoza, J., et al., Food-reward signalling in the suprachiasmatic clock. J Neurochem, 2010. 112(6): p. 1489-99.

47. Flores, D.E., C.N. Bettilyon, and S. Yamazaki, Period-independent novel circadian oscillators revealed by timed exercise and palatable meals. Sci Rep, 2016. 6: p. 21945.

48. Webb, I.C., M.C. Antle, and R.E. Mistlberger, Requlation of circadian rhythms in mammals $\underline{\text { by }}$ behavioral arousal. Behav Neurosci, 2014. 128(3): p. 304-25.

49. Voigt, J.P. and $\underline{H}$. Fink, Serotonin controlling feeding and satiety. Behav Brain Res, 2015. 277: p. 14-31.

50. Belle, M.D., et al., Acute suppressive and long-term phase modulation actions of orexin on the mammalian circadian clock. J Neurosci, 2014. 34(10): p. 3607-21.

51. Mahler, S.V., K.S. Smith, and K.C. Berridge, Endocannabinoid hedonic hotspot for sensory pleasure: anandamide in nucleus accumbens shell enhances 'liking' of a sweet reward. Neuropsychopharmacology, 2007. 32(11): p. 2267-78.

52. Hanlon, E.C., et al., Circadian rhythm of circulating levels of the endocannabinoid 2arachidonoylglycerol. J Clin Endocrinol Metab, 2015. 100(1): p. 220-6.

53. Acuna-Goycolea, C.., K. Obrietan, and A.N. van den Pol, Cannabinoids excite circadian clock neurons. J Neurosci, 2010. 30(30): p. 10061-6.

54. DiFeliceantonio, A.G. and K.C. Berridge, Dorsolateral neostriatum contribution to incentive salience: opioid or dopamine stimulation makes one reward cue more motivationally attractive than another. Eur J Neurosci, 2016. 43(9): p. 1203-18.

55. Vansteensel, M.J., et al., The opioid fentanyl affects light input, electrical activity and Per gene expression in the hamster suprachiasmatic nuclei. Eur J Neurosci, 2005. 21(11): p. 2958$\underline{66 .}$

56. Prosser, R.A. and H.E. Bergeron, Leptin phase-advances the rat suprachiasmatic circadian clock in vitro. Neurosci Lett, 2003. 336(3): p. 139-42.

57. Yannielli, P.C., et al., Ghrelin effects on the circadian system of mice. J Neurosci, 2007. 27(11): p. $2890-5$.

58. Hakansson, M.L., et al., Leptin receptor immunoreactivity in chemically defined target neurons of the hypothalamus. J Neurosci, 1998. 18(1): p. 559-72.

59. Zigman, J.M., et al., Expression of ghrelin receptor $m R N A$ in the rat and the mouse brain. $\underline{J}$ Comp Neurol, 2006. 494(3): p. 528-48.

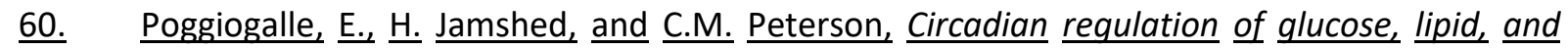
energy metabolism in humans. Metabolism, 2018. 84: p. 11-27.

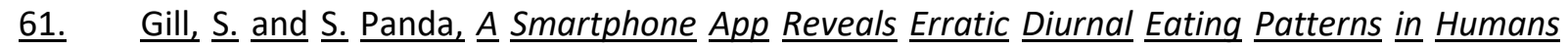

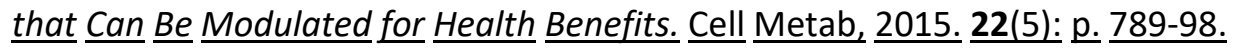


62. Vetter, $\underline{\text { C. }}$ and F. Scheer, Circadian Biology: Uncoupling Human Body Clocks by Food Timing. Curr Biol, 2017. 27(13): p. R656-R658.

63. McHill, A.W., et al., Later circadian timing of food intake is associated with increased body fat. Am J Clin Nutr, 2017. 106(5): p. 1213-1219.

64. Haus, E., et al., Risk of obesity in male shift workers: $\underline{A}$ chronophysiological approach. Chronobiol Int, 2016. 33(8): p. 1018-36.

65. Roenneberg, T., et al., Social jetlag and obesity. Curr Biol, 2012. 22(10): p. 939-43.

66. Bandin, $C_{.}$et al., Meal timing affects glucose tolerance, substrate oxidation and circadianrelated variables: A randomized, crossover trial. Int J Obes (Lond), 2015. 39(5): p. 828-33.

67. Qian, J.., et al., Ghrelin is impacted by the endogenous circadian system and by circadian misalignment in humans. Int $\mathrm{J}$ Obes (Lond), 2018.

68. Sutton, E.F., et al., Early Time-Restricted Feeding Improves Insulin Sensitivity, Blood Pressure, and Oxidative Stress Even without Weight Loss in Men with Prediabetes. Cell Metab, 2018. 27(6): p. 1212-1221 e3.

69. Versteeg, R.I., et al., Timing of caloric intake during weight loss differentially affects striatal dopamine transporter and thalamic serotonin transporter binding. FASEB J, 2017. 31(10): p. 4545-4554.

70. Volkow, N.D., G.J. Wang, and R.D. Baler, Reward, dopamine and the control of food intake: implications for obesity. Trends Cogn Sci, 2011. 15(1): p. 37-46.

71. Sheward, W.J., et al., Entrainment to feeding but not to light: circadian phenotype of VPAC2 receptor-null mice. $\mathrm{J}$ Neurosci, 2007. 27(16): p. 4351-8.

72. Maywood, E.S., et al., Disruption of peripheral circadian timekeeping in a mouse model of

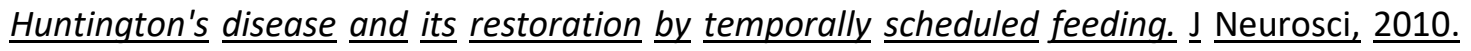
30(30): p. 10199-204.

73. Abe, $\underline{H .}, \underline{\mathrm{S}}$. Honma, and K. Honma, Daily restricted feeding resets the circadian clock in the suprachiasmatic nucleus of $\underline{\text { CS }}$ mice. Am J Physiol Regul Integr Comp Physiol, 2007. 292(1): p. R607-15.

74. Johnston, J.D., et al., Circadian Rhythms, Metabolism, and Chrononutrition in Rodents and Humans. Adv Nutr, 2016. 7(2): p. 399-406.

75. Takahashi, J.S., Molecular components of the circadian clock in mammals. Diabetes Obes Metab, 2015. 17 Suppl 1: p. 6-11.

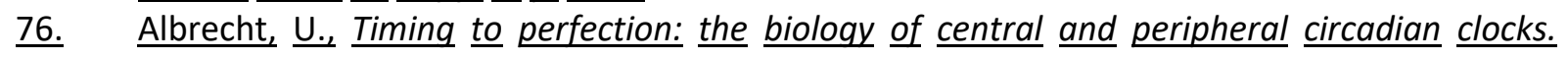
Neuron, 2012. 74(2): p. 246-60.

77. Schmidt, T.M., et al., Melanopsin-positive intrinsically photosensitive retinal ganglion cells: from form to function. J Neurosci, 2011. 31(45): p. 16094-101.

78. Lazzerini Ospri, L., G. Prusky, and S. Hattar, Mood, the Circadian System, and Melanopsin Retinal Ganglion Cells. Annu Rev Neurosci, 2017. 40: p. 539-556.

79. Stokkan, K.A., et al., Entrainment of the circadian clock in the liver by feeding. Science, 2001. 291(5503): p. 490-3.

80. Yamazaki, S., et al., Resetting central and peripheral circadian oscillators in transqenic rats. Science, 2000. 288(5466): p. 682-5.

81. Wuarin, J. and U. Schibler, Expression of the liver-enriched transcriptional activator protein DBP follows a stringent circadian rhythm. Cell, 1990. 63(6): p. 1257-66.

82. Lamia, K.A., K.F. Storch, and C.J. Weitz, Physiological significance of a peripheral tissue circadian clock. Proc Natl Acad Sci $\underline{\cup} \underline{S} \underline{A}$, 2008. 105(39): p. 15172-7.

83. Pulimeno, $\underline{P_{.}}$et al., Autonomous and self-sustained circadian oscillators displayed in human islet cells. Diabetologia, 2013. 56(3): p. 497-507.

84. Perelis, $\mathrm{M}_{\text {., }}$ et al., Pancreatic beta cell enhancers requlate rhythmic transcription of genes controlling insulin secretion. Science, 2015. 350(6261): p. aac4250.

85. Marcheva, B., et al., Disruption of the clock components CLOCK and BMAL1 leads to hypoinsulinaemia and diabetes. Nature, 2010. 466(7306): p. 627-31. 
86. Martino, T.A. and M.E. Young, Influence of the cardiomyocyte circadian clock on cardiac physiology and pathophysiology. $\mathrm{J}$ Biol Rhythms, 2015. 30(3): p. 183-205.

\section{Figure legends}

Figure 1. Daily and circadian rhythms of eating behavior are regulated centrally by the

Metabolic-Hedonic-Circadian network. Food intake is controlled by specific brain sites driving the metabolic and hedonic pathways of eating in the hypothalamus and mesocortico limbic system respectively. Accompanying these two pathways, the suprachiasmatic (SCN) clock, and its molecular machinery (Box 1), dictates the time of both, the need and pleasure of eating. The SCN receives direct projections from retinal ganglion cells allowing the synchronization to the light-dark cycle (LD). Thus, daily hedonic and metabolic eating is time-controlled, and synchronized to the LD cycle (Box 2), giving a robust food intake at day for diurnal species (including humans) and at night for nocturnal animals (e.g., rats, mice, hamsters). The SCN sends neural and paracrine timing signals to both hedonic and metabolic centers of food intake to impose rhythms of eating. Interestingly, spite that the functional clockwork of the SCN between diurnal and diurnal species is similar, food intake is opposite during the 24h-day, suggesting that the time of eating behavior may reside somewhere in these brain feeding centers (i.e., metabolic, hedonic).

Figure 2. Time eating behavior drives brain and peripheral clocks and circadian in the brain and periphery. TRE aligned with the active phase of the organism (at day for diurnal species, and at night for nocturnal) favors synchronization and improves physiology 
and health. However, when time of feeding is misaligned to the sleep-wake cycle, metabolic, behavioral and physiological consequences emerge (e.g., body weight gain, glucose metabolism dysregulation). TRE coupled to a hypocaloric condition, or with a strong hedonic background entrains the main SCN clock. The SCN projects to the metabolic and hedonic brain feeding centers, and to peripheral oscillators via the autonomous nervous system (ANS). On the other way around the SCN is innervated by these structures and peripheral hormones which will affect the clock in food entrainment (B) At the SCN level, feeding time might affect the physiology and molecular properties of the clock through the release of diverse neurotransmitters and the activation of diverse receptors and the intercellular pathways In addition, peripheral hormones (e.g., ghrelin, leptin) synchronized by feeding time may act directly in their receptors localized in the SCN and other brain circadian clocks to potentiated food synchronization. (C) Representative actogram of the behavioral circadian rhythm (in constant darkness, DD) of wheel running activity of a mouse synchronized to daily palatable meal (chocolate) intake without food restriction (red vertical line). 5-HT, serotonin; 5-HT1A, serotonin 1A receptor; $\mathrm{Ca}^{2+}$, calcium; CaMKK, Calcium/calmodulin-dependent protein kinase; CB1, cannabinoid 1 receptor; CREB, cAMP-responsive element-binding protein; D1R, dopamine 1 receptor; DA, dopamine; $\delta$-R, delta-opioid receptor E-box, enhancer box; ENDO, endocannabinoids; ERK, extracellular signal-regulated kinase; GABA $A_{A}, G A B A_{A}$ receptor; GHSR, Growth hormone secretagogue receptor; GSK3, Glycogen synthase kinase3; JAK, Janus Kinase; LepR, leptin receptors; MAPK, mitogen-activated protein kinase; $\mu$-R, $\mu \mathrm{u}$-opioid receptor; NPY, neuropeptide Y; NPY1R, neuropeptide Y1 receptor; ORX, orexin; OX-1R, orexin 1 receptor; OXM, oxyntomodulin; PKA, protein kinase A; SIRT1, sirtuin 1; STAT-3, signal transducer and activator of transcription.

. 


\section{Box 1. Molecular clockwork}

$\underline{622}$

$\underline{623}$

$\underline{624}$

$\underline{625}$

$\underline{626}$

$\underline{627}$

$\underline{628}$

$\underline{629}$

$\underline{630}$

$\underline{631}$

$\underline{632}$

$\underline{633}$

$\underline{634}$

$\underline{635}$

The molecular timekeeping of the SCN is dependent on the clock genes Clock and Bmall, two transcription factors which are able to dimerize and bind to the E-box of other genes, such as Period (Perl-3) and Cryptochrome (Cryl-2). PER-CRY, have the capacity to enter back to the nucleus to inhibit the activity of CLOCK-BMAL1, and thus their own transcription [75]. The final result is the oscillatory activity (around the 24h) of these genes (excluding Clock) in the SCN, with peaks of activity during the light period for Perl-3 and Cryl-2 genes, and Bmall with a peak during the dark period. Another group of genes oscillating in a circadian manner and dependent on the CLOCK-BMAL1 dimer are the nuclear hormone receptors Rev$\operatorname{Erb}(\alpha / \beta)$ and $\operatorname{Ror}(\alpha, \beta$, and $\gamma)$. These genes induce the circadian rhythmicity of Bmall: Rev$E r b \alpha$ represses Bmall during the day while Ror $\beta$ activates it at night. Finally, clock-output or clock-controlled genes (CCG) from the SCN signal the rest of the brain and body to give the timing cue required, and keep circadian synchrony in the whole body; vasopressin ( $A v p)$ is the principal SCN output signal. Moreover, other SCN output signals are humoral or paracrine. The peptides transforming growth factor alpha (TGF $\alpha$ ), prokineticin 2 (PK2) and cardiotrophin-like cytokine (CLC) participate as temporal SCN signals for the circadian control of behavior [6]. The sophisticated clockwork enginery in the SCN has been also described in other tissues, such as the liver, heart, lung, kidney, pancreas and adipose tissue; thus, despite the initial identification of a single body pacemaker in the brain, now it is well 
known that several organs in the whole body contain a circadian clock indispensable for the rhythmic functions accorded to each tissue [76].

\section{Box 2. Light-synchronization of circadian clocks}

The SCN is mainly synchronized to the day-night alternation [6]; Light is perceived in the eye by a particular layer of ganglion neurons containing the pothopigment melanopsin, also called intrinsically photosensitive retinal ganglion cells (ipRGCs). These cells project to the SCN (in the ventral neurons), through the monosynaptic retinohypothalamic tract (RHT), for entrainment to the LD cycle [77]. At cellular level light signaling promotes an increase in the influx of $\mathrm{Ca}^{2+}$ in postsynaptic SCN cells, then an activation of protein kinases and the cAMP response element binding protein (CREB), which finally binds in the promoters of Perl-2 genes, leading to the resetting of the phase and/or entrainment of the clock.

Moreover, since ipRGCs connect with other brain structures in the hypothalamus (e.g., lateral hypothalamus, preoptic area) and epithalamus (perihabenular nucleus), light is able to modulate specific behaviors (e.g., sleep, feeding, motivation) [78]. Thus, the LD cycle may entrain brain circadian clocks and behavior directly and/or indirectly via the SCN. For peripheral oscillators, however, LD signals first entrain the SCN, and then the clock uses hormonal and neuronal pathways, behavioral (e.g., food intake, locomotor activity) or temperature cues to transmit the solar time information to the whole body [37].

\section{Box 3. Peripheral circadian oscillators}

Several years have passed already from the first discovery on the expression of clock genes oscillating in the liver $[31,79,80]$. The liver is a peripheral circadian clock which plays an important role in physiology and metabolism. Interestingly, the first reports on the circadian activity of liver were made by serendipity by the group of Prof. Ueli Schibler. In the interest of studying the albumin gene, authors isolated the transcription factor D-element Binding Protein (DBP) with the surprising results that accumulation of $D b p$ in the liver is a hundred times more at night than day, and independent of the SCN and/or rhythmic hormone release [81]. How important is the rhythmic gene expression in the liver? A specific Bmall deletion in the liver clock alters glucose metabolism inducing a decrease in the levels of circulating glucose in blood at daytime, thus, altering the endogenous rhythm of glucose production necessary for the metabolic challenges during sleep/fasting conditions [82].

In the pancreas, $\beta$-cells exhibit rhythmic expression of circadian genes which induces diurnal insulin secretion $[83,84]$. Considerably, clock capacity in islet pancreas cells is dependent on 
clock genes, since when one of the principal actors of the molecular clockwork, such as Clock or Bmall is lacking, the insulin secretion is altered, leading to possible development of diabetes [85]. Thus, the pancreatic circadian clock locally regulates insulin release in time; a circadian dysfunction of it leads to metabolic pathology.

In the cardiovascular system, a circadian clock regulates daily changes in blood pressure (BP) and heart rate (HR). Clock genes and proteins (Bmal1, Per2, Rev-Erbo) and clock-output genes $(D b p)$ are rhythmically expressed in the heart and these rhythms persist ex vivo, indicating the presence of an autonomous circadian clock within cardiac cells [86]. Moreover, global and specific mutations of the BMAL1 clock gene lead in alterations in of cardiovascular functions (BP, HR), bradycardia and depressed systolic function. In humans, cardiovascular incidents (myocardial infarction) show a higher frequency in the morning (6:00-12:00 a.m.), which can be correlated to the circadian variations of cardiac functions (BP, HR, blood consistence) [87]. The clock in the cardiovascular system is synchronized by the SCN via the autonomous nervous system [88], therefore, a misalign between the SCN and the Heart clock by circadian disruptions (jet-lag, shift work) may potentiate the risk for cardiovascular diseases [87].

\section{Box 4. Extra-SCN brain circadian oscillators}

In the brain, extra-SCN structures have been identified as circadian oscillators with selfsustained ability and synchronized by the SCN. These are the olfactory bulb (OB) [89] and the lateral habenula $(\mathrm{LHb})[90,91]$; both are good examples of self-sustained oscillators, independent of, but entrained by the SCN, and dependent on clock genes. The clock in the OB controls the circadian rhythms in odor detection, and this behavior might be relevant in nature for foraging and optimizing the quality of food sources. The LHb has been revealed as a relevant structure for the control of monoamines. Moreover, it has been implicated in the regulation of palatable and caloric food intake $[23,24]$. In the hypothalamus, beyond the $\mathrm{SCN}$, the arcuate (ARC) and dorsomedial (DMH) nuclei have circadian activity in the firing rate and clock gene expression, and these are importantly affected by feeding status in mice [92]. However, these have been classified as slave circadian oscillators. 

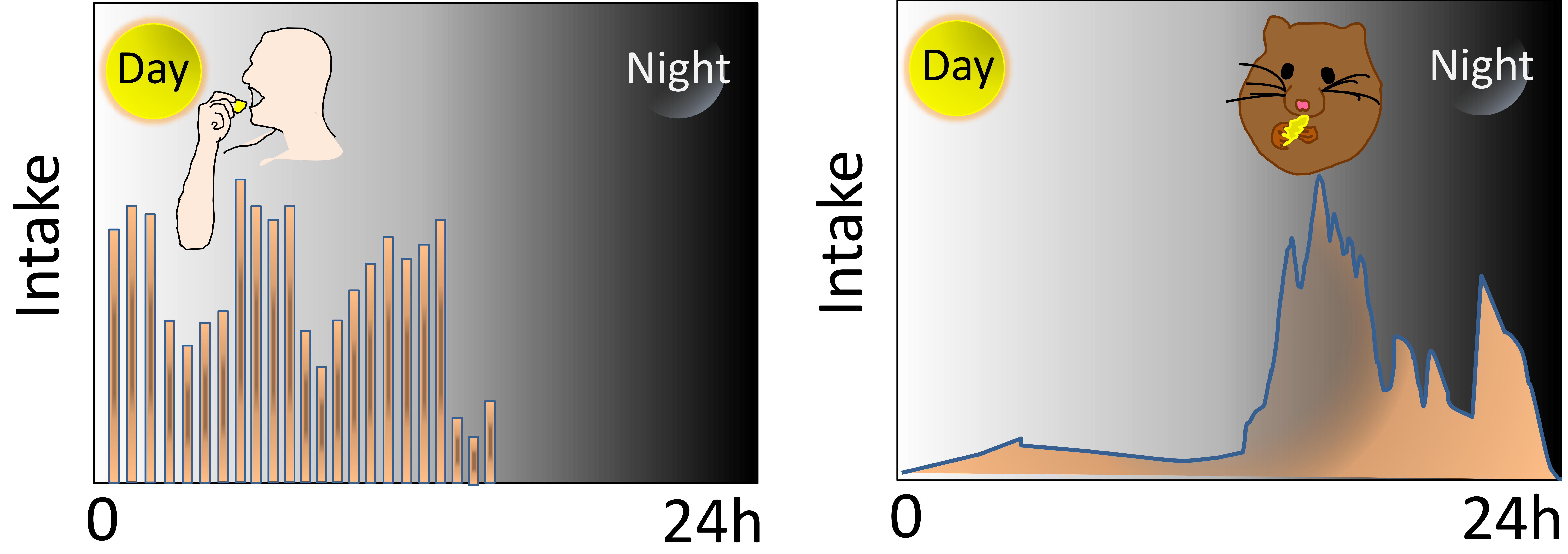

DAY-NIGHT CYCLE

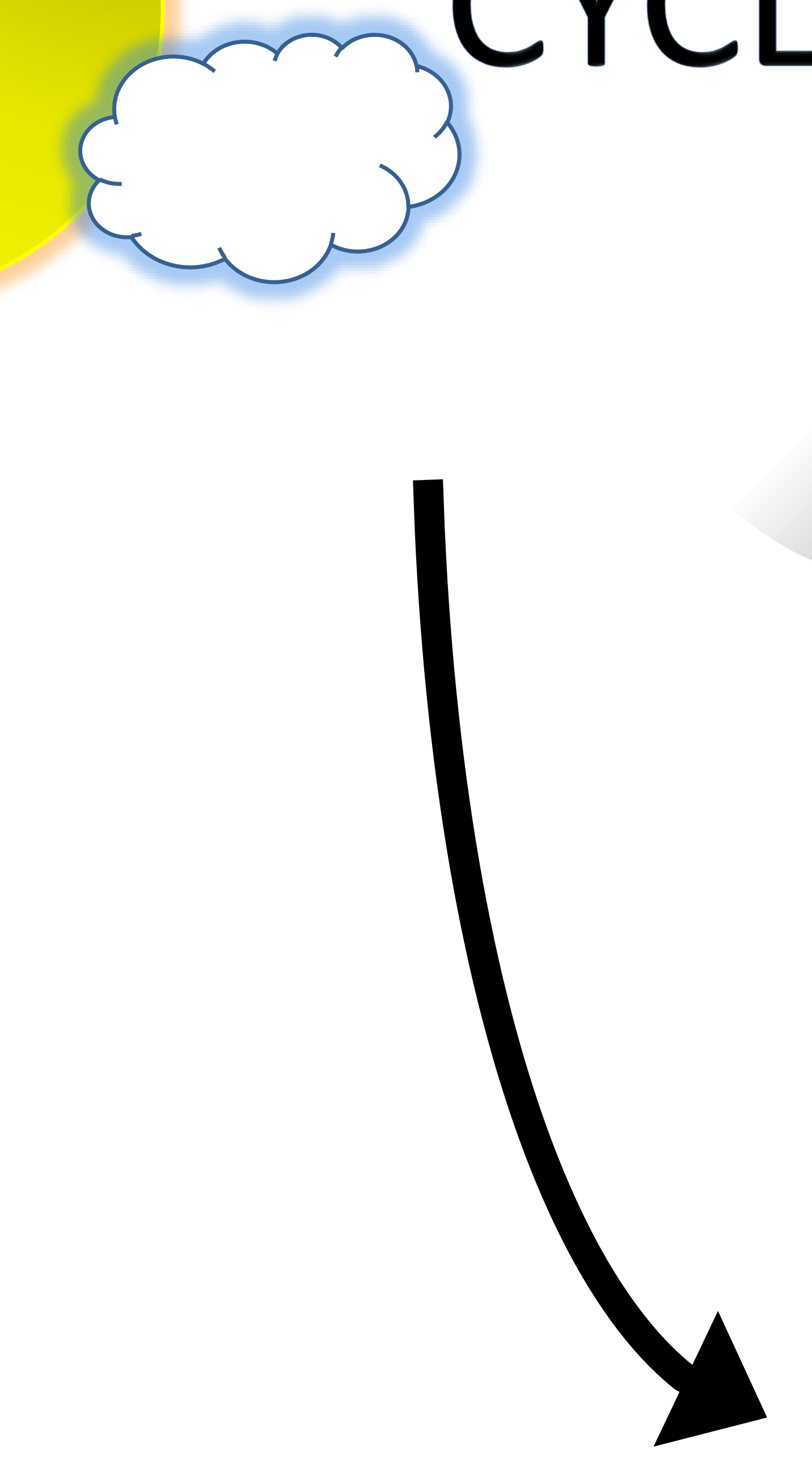

Metabolic

Hypothalamus

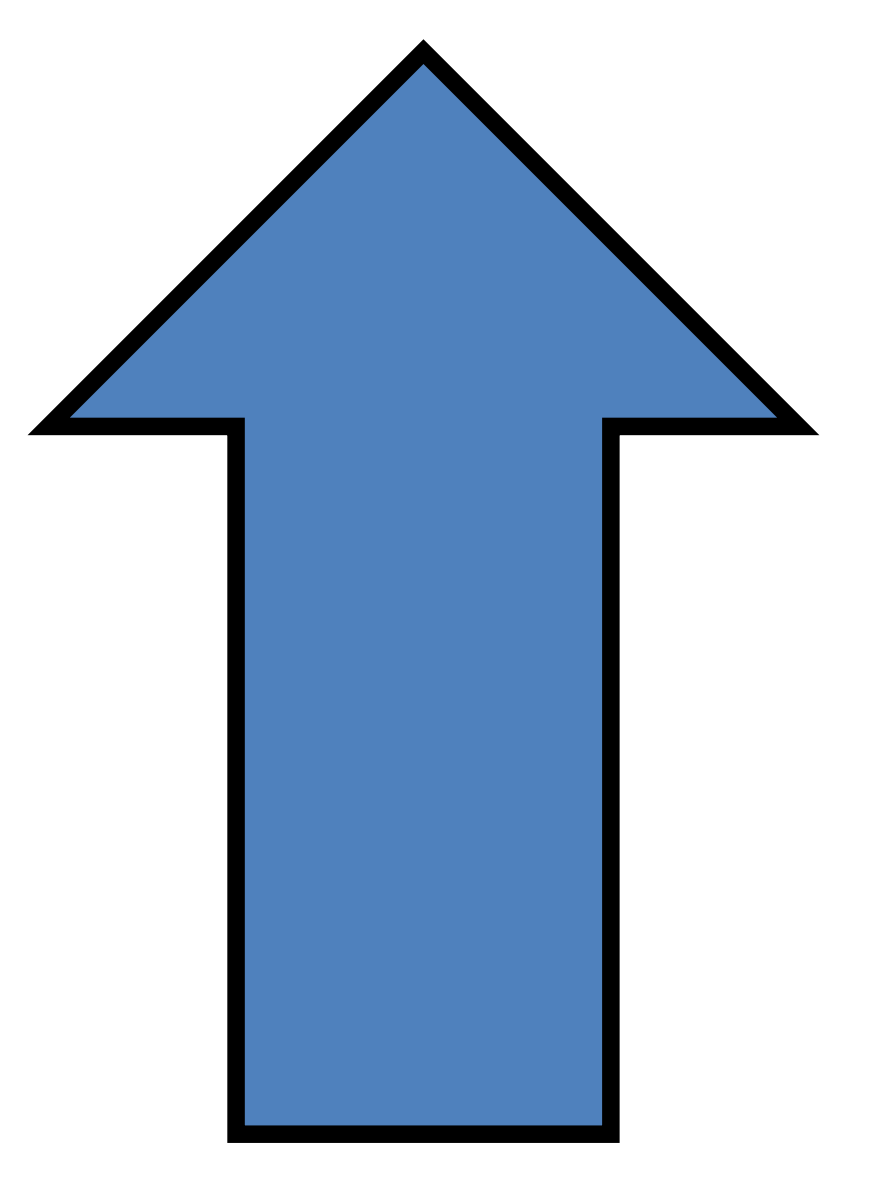

$24 \mathrm{~h}$

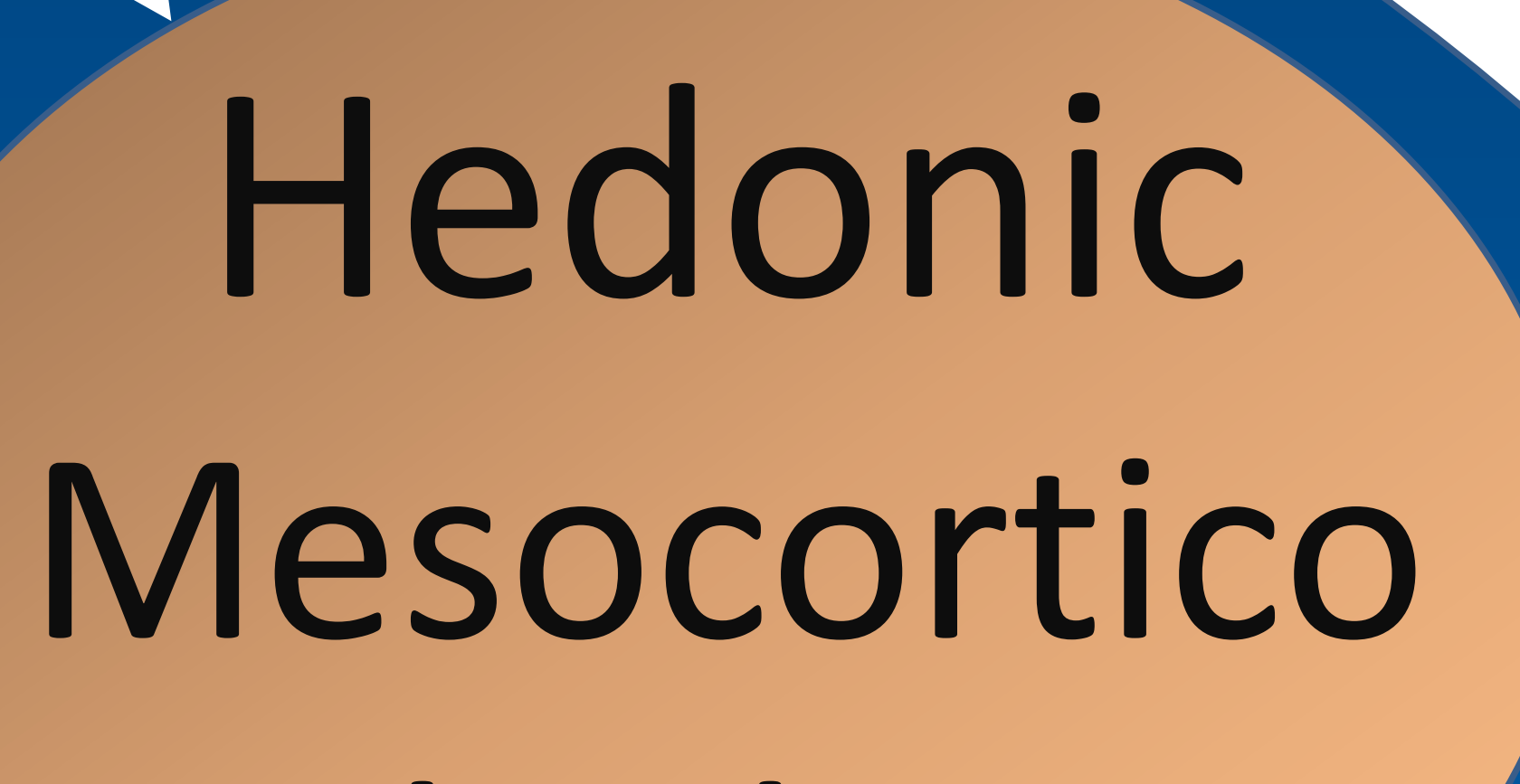
limbic

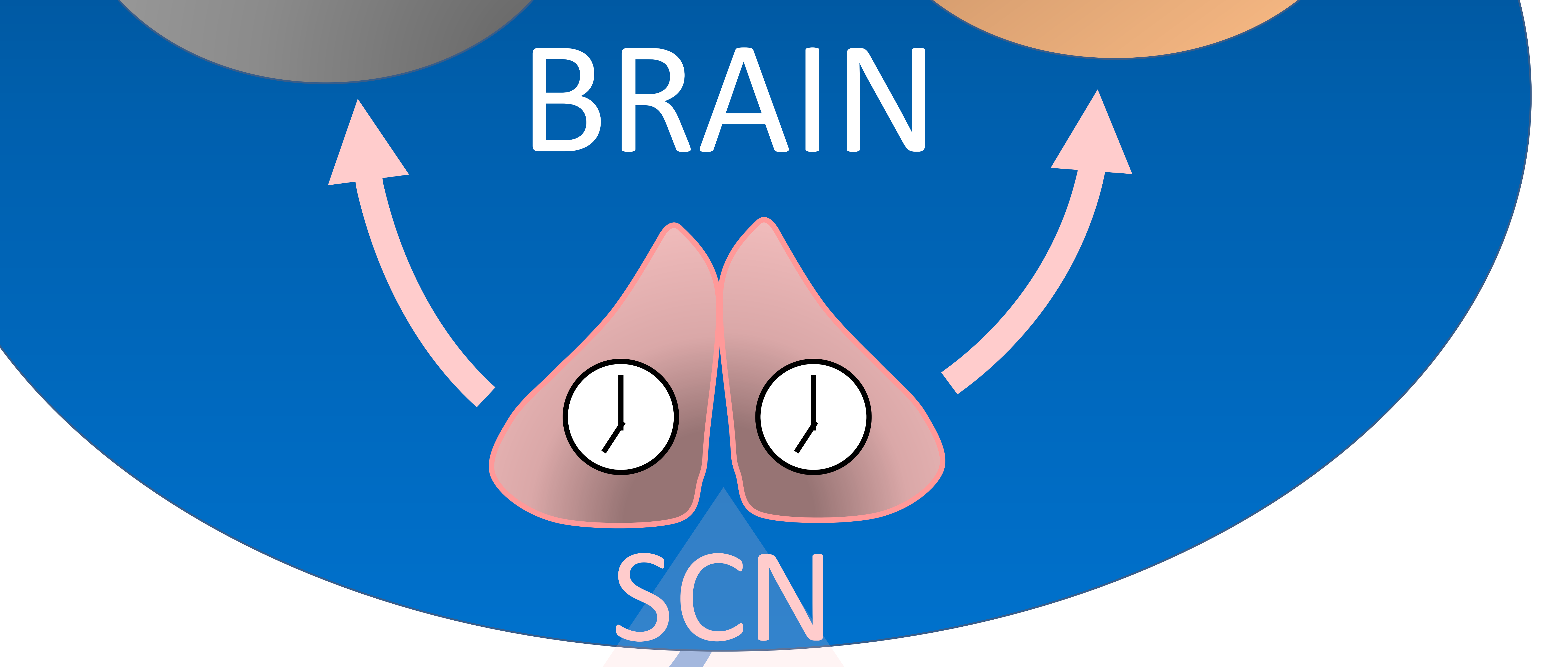

EYE

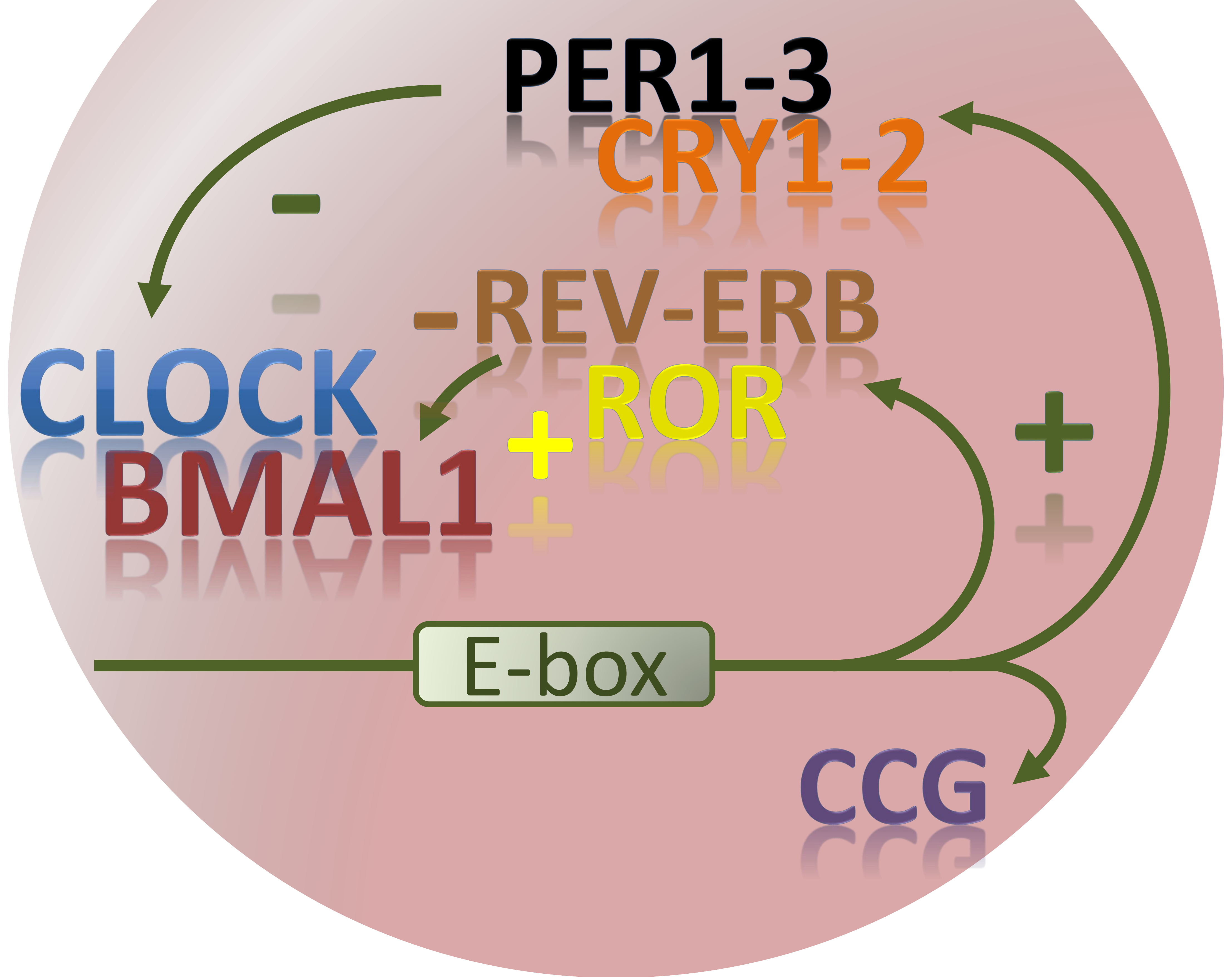


O O 